Scand 2011;124(Suppl 192):83-91). Field defects are bilateral and more pronounced nasally. Reported estimates of pVFDs are $30-50 \%$ in adults and $20 \%$ in children. Risk increases with increased dosage and duration of therapy. Vision screening is recommended at baseline, every 3 months, and at 3-6 months after discontinuation of therapy.

\title{
SEIZURE-INDUCED MIOSIS
}

Researchers at University of Southampton, UK report an infant with focal seizures secondary to cortical dysplasia who presented at age 4 months with bilateral pinpoint pupils as first feature of right-sided clonic seizure. EEG recording during seizures showed ictal spiking over the left central region. ECG did not show bradycardia, and there were no signs of parasympathetic activation. MRI, obtained after relapse at 13 months of age with 30 seizures daily involving the right upper limb, revealed left frontal cortical dysplasia. A cortical electrographic grid and intracranial EEG demonstrated ictal fast spike activity within the left centroparietal region, over the middle parietal gyrus. The irritative zone was located at the margin and across the area of dysplasia. The child remained seizure-free at 3 years following excision of the lesion. The origin of ictal miosis is considered secondary to activation of a cortical pupillary constrictor zone as

opposed to activation of the parasympathetic nervous system through the subcortical central autonomic network, usually resulting in mydriasis. (Sadek A-R, Kirkham F, Barker S, Gray WP, Allen D. Seizure-induced miosis. Epilepsia Dec 2011;52(12):e199e203). (Respond: Dr Ahmed-Ramadan Sadek, Division of Clinical Neurosciences, School of Medicine, University of Southampton, Tremona Road, Southampton SO16 6YD, UK. E-mail: a.sadek@soton.ac.uk).

COMMENT. Bilateral pupillary dilatation preceding, during, or after generalized convulsive or nonconvulsive seizures is common, but ictal pupillary miosis is rare. Autonomic disturbances during seizures (alterations in heart rate, blood pressure, gastrointestinal function) result from seizures within the largely subcortical and brainstem central autonomic network. The UK study demonstrates ictal miosis originating in the middle parietal gyrus juxtaposed by the superior anastomatic vein of Trolard, within the cortical central parietal region.

\section{MITOCHONDRIAL HYPERMETABOLIC NEURONS IN EPILEPSY}

Researchers at the University of Calgary and Alberta Children's Hospital, Canada studied histochemically frozen sections of 10 brain resections from 7 epileptic children (ages 2 months to 17 years). None had mitochondrial disease; 1 had tuberous sclerosis and 2 hemimegalencephaly. Preop EEG and intra-operative ECoG defined foci refractory to AEDs. Increased mitochondrial respiratory chain enzymes were demonstrated in individual neurons of hippocampal and neocortex epileptic foci. These intensely staining neurons may indicate functional hypermetabolism and represent a metabolic marker of an epileptic focus. Alpha-B-crystallin was also strongly reactive, demarcating the epileptic focus as a wide field. (Sarnat HB, Flores-Sarnat L, Hader W, Bello-Espinosa L. Mitochondrial "hypermetabolic" neurons in paediatric epileptic foci. Can J Neurol Sci 
2011;38:909-917). (Respond: Dr HB Sarnat, Alberta Children's Hospital, 2888 Shaganappi Trail NW, Calgary, Alberta T3B 6A8, Canada).

COMMENT. Metabolic tissue markers of epileptic foci help define a total excision of brain tissue in the surgical management of refractory epilepsies. Increased mitochondrial respiratory chain enzymes and/or intense a-B-crystallin reactivity at the periphery of resected brain tissue may indicate incomplete removal of a focus and an increased risk of seizure recurrence. (Sarnat HB, Flores-Sarnat L. Can J Neurol Sci 2009;36:566-574). These novel tissue markers add immunohistochemistry to EEG telemetry, ECoG, and MRI as tools to determine the extent of resection of epileptic foci of brain tissue.

Epileptic networks in focal cortical dysplasia revealed by EEG and fMRI. Of 23 patients with focal cortical dysplasia and epilepsy undergoing presurgical evaluation with intracranial EEG (icEEG) and simultaneous EEG-fMRI, 12 had interictal discharges (IED) during recording, and 11 of 12 had IED-related hemodynamic changes. The fMRI results were concordant with the seizure onset zone (SOZ) on icEEG in 5 of 11 patients. Four of 5 had $>50 \%$ reduction in seizure frequency following resective surgery. The remaining 6 had discordant IED-related fMRI signal change. Five of 6 had a poor surgical outcome. Discordant regions of IED-related hemodynamic change are associated with a widespread seizure onset zone and poor surgical outcome. (Thornton R et al. Ann Neurol Nov 2011;70(5):822-837). (Response. Louis Lemieux PhD, Institute of Neurology, London, UK. E-mail: Louis.lemieux@ucl.ac.uk).

\section{HEADACHE DISORDERS}

\section{HEADACHE POST-TRAUMATIC BRAIN INJURY}

Researchers at University of Washington, Seattle, and Children's Hospital of Philadelphia, PA conducted a prospective cohort study of the prevalence of headache 3 and 12 months after traumatic brain injury (TBI) in children ages 5 to 17 years. Followup interviews with parents or adolescents found the prevalence of headache 3 months after injury was significantly higher after mild TBI than in controls after arm injury overall (43\% vs 26\%), in adolescents, and in girls. It was also higher after moderate/severe TBI in younger children. At 12 months after injury, TBI was not associated with a significantly increased frequency of headaches, but girls with mild TBI reported serious headache more often than controls. (Blume HK, Vavilala MS, Jaffe KM, et al. Headache after pediatric traumatic brain injury: A cohort study. Pediatrics 2012;129:1-9). (Respond: Heidi K Blume MD, MPH, Division of Pediatric Neurology, University of Washington, Seattle Children's Hospital and Research Institute, Seattle, WA 98105. E-mail: Heidi.blume@seattlechildrens.org).

COMMENT. Girls and adolescents are at highest risk of headache in the months after mild TBI. Younger children also suffer long-term headache after moderate/severe TBI. Posttraumatic headache after mild TBI shares similarities with migraine and tension-type headache. Migraine headache in children may be precipitated by head 